\title{
The long-term outcomes of early repolarization pattern and incidence of early repolarization syndrome in a population-based cohort study
}

\author{
songkeat yodteerug ${ }^{1}$, Prin Vathesatogkit ${ }^{1}$, Pattara Rattanawong ${ }^{2}$, Tachapong Ngarmukos ${ }^{1}$, \\ Sirin Apiyasawat ${ }^{1}$, and Pakorn Chandanamattha ${ }^{1}$ \\ ${ }^{1}$ Mahidol University Faculty of Medicine Ramathibodi Hospital \\ ${ }^{2}$ University of Hawaii Internal Medicine Residency Program
}

September 23, 2020

\begin{abstract}
Background : Early repolarization pattern (ERP) is associated with long-term cardiovascular death. However, the incidence of early repolarization syndrome ( ERS) has never been studied in a general population-based cohort study. Purpose : To determine the prevalence and long-term outcome of ERP as well as the incidence of ERS in a general population-based cohort study. Methods : Participants from the electricity generating authority of Thailand (EGAT) study during 1997 to 2015 were included. The outcomes included cardiovascular death,deaths due to acute myocardial infarction, SCD, and all-cause mortality. A Cox- proportional hazard model was used to determine the association between ERP and the outcomes. Results: 2,689 participants with completed ECGs and risk factor profiles were included for the analysis. Mean follow up duration was 11.2 \pm 6.7 years. There were 444 participants with baseline ERP. There were 566 deaths during the follow-ups; of these, 21 were SCD including 6 ERS. The prevalence and incidence of ERS in our study was $0.22 \%$ and 0.20 per 1000 person-year. Overall, ERP was not associated with an increased risk of all-cause mortality (hazard ratio $[\mathrm{HR}]=1.04 ; 95 \%$ confidence interval $[\mathrm{CI}]: 0.81$ to $1.34 ; \mathrm{p}=0.75)$. However, ERP was associated with an increased risk of all-cause mortality in the population [?]55 years old $(\mathrm{HR}=2.36 ; 95 \% \mathrm{CI}: 1.47-3.77 ; \mathrm{p}<0.01)$. Conclusion : The prevalence of ERP in the Thai population was higher than the prevalence in other countries in Asia. Our study supports previous studies that ERP is associated with a long-term increased risk of all-cause mortality in the young-middle age population.
\end{abstract}

\section{Hosted file}

proof Early Repo manuscipt 25Aug2020.pdf available at https://authorea.com/users/361256/ articles/482719-the-long-term-outcomes-of-early-repolarization-pattern-and-incidence-ofearly-repolarization-syndrome-in-a-population-based-cohort-study 